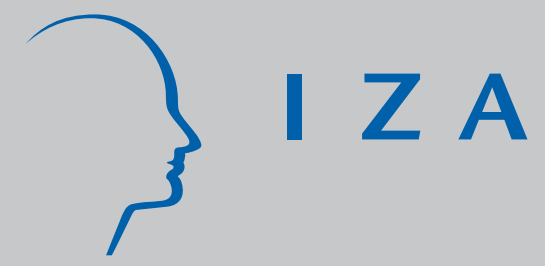

IZA DP No. 4234

Network Formations among Immigrants and Natives

Gil S. Epstein

Odelia Heizler (Cohen)

June 2009 


\title{
Network Formations among Immigrants and Natives
}

\author{
Gil S. Epstein \\ Bar-llan University, CReAM \\ and IZA \\ Odelia Heizler (Cohen) \\ Hebrew University of Jerusalem \\ and IZA \\ Discussion Paper No. 4234 \\ June 2009 \\ IZA \\ P.O. Box 7240 \\ 53072 Bonn \\ Germany \\ Phone: +49-228-3894-0 \\ Fax: +49-228-3894-180 \\ E-mail: iza@iza.org
}

\begin{abstract}
Any opinions expressed here are those of the author(s) and not those of IZA. Research published in this series may include views on policy, but the institute itself takes no institutional policy positions.

The Institute for the Study of Labor (IZA) in Bonn is a local and virtual international research center and a place of communication between science, politics and business. IZA is an independent nonprofit organization supported by Deutsche Post Foundation. The center is associated with the University of Bonn and offers a stimulating research environment through its international network, workshops and conferences, data service, project support, research visits and doctoral program. IZA engages in (i) original and internationally competitive research in all fields of labor economics, (ii) development of policy concepts, and (iii) dissemination of research results and concepts to the interested public.
\end{abstract}

IZA Discussion Papers often represent preliminary work and are circulated to encourage discussion. Citation of such a paper should account for its provisional character. A revised version may be available directly from the author. 
IZA Discussion Paper No. 4234

June 2009

\section{ABSTRACT}

\section{Network Formations among Immigrants and Natives}

In this paper we examine possible network formations among immigrants and natives with endogenous investment. We consider a model of a network formation where the initiator of the link bears its cost while both agents benefit from it. We present the model by considering possible interactions between immigrants and the new society in the host country: assimilation, separation, integration and marginalization. The paper highlights different aspects of immigrants' behavior and their interaction with the members of the host country (society) and their source country (society). We found that when the stock of the immigrants in the host country increases, the immigrants' investment in the middlemen increases and the natives may bear the cost of link formation with the middlemen.

JEL Classification: D85, J15, F22, A14

Keywords: network formations, social networks, assimilation and separation

Corresponding author:

Odelia Heizler (Cohen)

Department of Agricultural Economics and Management

The Hebrew University

P.O. Box 12

Rehovot 76100

Israel

E-mail: ocohen@biu.013.net.il 


\section{Introduction}

Consider a new immigrant in an unfamiliar environment. The migrant does not know the foreign location, the local language and the customs of the host society. The migrant needs assistance in order to become familiar with the new environment and to find accommodation and a job. If in the host country there are migrants from the same origin, who arrived earlier in time, they will most probably be able to provide the new migrant ethnic networks, i.e. the necessary help in order to assimilate in the new location. The existence of this network has a great effect on the immigrants' choice of location.

Numerous studies have demonstrated that immigrants tend to settle in ethnic concentrations (see, for instance, Church and King, 1983; Bartel, 1989; Borjas, 1998). Once the first immigrant settles in a certain location, future immigrants are no longer indifferent about destinations. As the stock of immigrants increases, the cost of subsequent immigrants decreases (see Massey, 1987 and Carrington et al., 1996). This is because previous immigrants provide the new immigrant with social support, jobsearch assistance, help in finding accommodations and information on the alien environment and the local culture. Battu et al. (2004) shows theoretically and empirically that less assimilated ethnic unemployed workers are more likely to use their friends and family as their main method of search. However, they have less chance of finding a job using this method than whites and more assimilated ethnic unemployed workers who use formal job search methods (such as: advertisements, employment agencies, responding to newspaper advertisements, etc.).

In addition to the job-search assistance, the immigrants' concentration provides the settlers with "ethnic goods", the products and services which the immigrants do not share with the host population (e.g., saris, Chinese vegetables, kosher food, churches, mosques, temples, or synagogues). The cost of "ethnic goods" decreases with the number of immigrants who settle in the enclave and all the immigrants, old and new, benefit from the increase in its size (see Chiswick and Miller, 2005).

Residence in ethnic concentrations facilitates, at first, the assimilation process. However, studies show that it may have a negative effect on the success of adjustment. For example, Culter and Gleaser (1997) found that blacks who are located in more segregated areas have significantly worse success than blacks in less segregated areas. Borjas (2000) found that ethnic residential segregation hampers the 
process of economic assimilation. The reason for this negative effect on the assimilation process is that an increase in the immigrants' stock in the enclaves increases the competition for jobs and decreases the immigrants' wages (see Bauer Epstein and Gang, 2005; 2007). Furthermore, these enclaves create incentives for the immigrants not to learn the culture and language of the host country. A destination language deficiency reduces the income and harms assimilation (Chiswick 1978; Chiswick and Miller, 1996). Moreover, the existence of an ethnic enclave may cause xenophobia (Dustmann and Preston, 2000). Joining the local society requires the immigrant to invest effort and resources such as investment in learning the local language and customs.

The residence of an immigrant in a segregated area affects the interaction with the dominant (local) society. Berry (1997) points to four possible models for interaction between the immigrants and the new society. Assimilation takes place when individuals break away from their cultural identity and seek daily interaction with the native culture. The immigrants are then assimilated in the host society and resemble the natives. Separation occurs when individuals place a value on retaining their original culture and wish to avoid interaction with the local culture. Integration occurs when the individuals are interested in maintaining the original culture in their daily interaction, in parallel with an attempt to become full participants in the new society. Marginalization occurs when the individuals fail to maintain interaction with both their original and local society thus pushing them to society’s margins. ${ }^{1}$

In this paper we set out a formal framework of possible network formations within the society and among different societies: natives and immigrants. One of the first decisions of the immigrant is with whom to maintain a link in the foreign country. In our model, the individual decides not only with regard to how many people he wishes to maintain a link and their identity (immigrants or natives), but also how much effort he invests in these links. Furthermore, we examined the possible interactions between the immigrants and the local society: assimilation, separation and integration.

\footnotetext{
${ }^{1}$ Recently, Echenique and Fryer (2007) presented a framework to measure the segregation between different groups. The analysis is based on social interactions of the members of a race.
} 
This study contributes to the existing literature in two distinct ways. Firstly, most of the existing literature assumes exogenous investment in the friendship link and homogenous agents, whereas in our model the investment in the friendship link is endogenous while we consider two different types of groups and secondly, we implement this model with regard to migration and present possible interactions between the immigrants and the new society.

The paper proceeds as follows: A background on network formation and a model of endogenous investment in friendship are presented in the next section. Implementation of this model to migration is presented in section 3. Section 4 contains concluding remarks.

\section{The model}

\subsection{Background}

There is a well-established and vast literature in economics and sociology on the role played by social networks in communicating valuable information. This information may include, among other things, job and accommodation options, business opportunities, stock market tips and the quality of products. For example, Montgomery (1991) presents a long list of research on the important role of social ties, such as friends and relatives, in job search outcomes. However, only few studies emphasize the role of social ties in job searches among minorities (see, for example, Holzer, 1987).

Social relationships come in many shapes and sizes, and there is no single model which encompasses them all together. Let us depict the main categories and characteristics of network formation in recent literature (for a comprehensive review see Jackson, 2003). Most of the existing network models of formation fall into one of two approaches: non-directed network and directed network. The former category takes place when two players are either connected or not and it is impossible that one is related to the second without the second being related to the first. The latter category occurs when one player may be connected to a second without the second being connected to the first.

Another characteristic of networks is who incurs the cost of link formation. In the majority of the studies both agents bear the cost for link formation, whereas in others the costs are borne only by the person who initiates the link (see Bala and Goyal, 2000a; 2000b). The literature on social networks focuses on stable and 
efficient networks and the relationship between these networks. For example Jackson and Wolinsky (1996) provide sufficient conditions for efficiency of different types of structures. The conditions are in terms of the cost and benefit of the link and the fact that the link requires the consent of both parties involved, but severance can be done unilaterally. Kim and Wong (2007) examine stability where single agent’s unilateral action is limited in affecting the network structure.

Most of the existing literature assumes that the agents are homogenous and the investment in the link formation is constant. However, there is an emerging literature which proposes a different approach to network formation. Goyal and Moraga Gonzales (2001) present a two-stage model where firms first establish links and then decide on their R\&D investment in every bilateral relationship. Brueckner (2006) considers a stochastic model of social networks where the level of investment in link formation is endogenous and the formation of a link between two agents requires a two-sided investment. Bloch and Dutta (2008) presents a model with an endogenous cost of the link formation focusing on two polar cases of agents' investments: perfect substitutes and perfect complements. They find that when the investments are perfect substitutes, the most efficient network is the symmetric star network, whereas when the investments are perfect complements, the symmetric line and cycle networks are both efficient and stable. Haller and Sarangi (2006) also develop a model of social network which enables different probabilities for different links basing on Bala and Goyal (2000b). Galeotti et al. (2006) present a model of network formation with heterogeneous players in respect to their benefits, as well as to the costs of forming links. They also deal with link formation using an insider-outsider models where the society is composed of distinct groups. Finally, Currarini et al. (2007) study a matching process of friendship-formation showing that the groups' sizes determine equilibrium outcome.

\subsection{Endogenous investment in friendship}

We consider a finite set of homogenous agents $N=\{1,2, \ldots i, \ldots, n\}$, where one individual joins the group each period. The group may be a religious group, a minority, political, sports, school fraternity, youth group, etc. The new individual, $i \in N$, has to decide how many and to what intensity he wishes to maintain his links. Decisions are made sequentially with people joining the group. Information is 
assumed to be complete, i.e. when a new agent joins the group he is aware of the links between the members of the group and thus his decision is based on this common knowledge. Each period, after the new individual has joined, all other individuals can update their decision by adding links, severing links or investing more or less efforts in the existent links.

We follow Watts (2001; 2002) and Jackson and Watts (2002) and consider myopic behavior, i.e. individuals do not forecast how their decision to add or sever a link could affect future decisions of others. As stated by these authors, such myopic behavior is a natural behavior in the context of large networks. In addition, the agents have limited information about the number of future agents who may or could join the group. For example, a member of a minority group doesn't know when the flow of immigrants to the destination country will cease, due to a reform in migration policy etc. Furthermore, Jackson (2003) presents a literature review of experiments that compare myopic versus forward-looking behavior in networks. He states that there is little evidence of forward-looking behavior even in environments designed to elicit it.

Following Bala and Goyal (2002a, 2002b), we present a non-cooperative model of network formation, assuming that the costs of link formation are incurred only by the agent who initiates the link, even though both agents benefit from the friendship. Hence, if one individual connects to another agent, he will bear the linking cost. ${ }^{2}$ Contrary to most of the existing literature, which assumes that the investment is a binary action (i.e., to form a link or not) and the cost is constant, we examine the scenario where investment is a continuous variable and is determined endogenously.

Denote the benefit from a friendship link by $u$ and the effort which is invested in agent number $j$ by agent number $i$ by $e_{i, j}$. The intensity (or the strength) of the relationship between $i$ and $j, p\left(e_{i, j}\right)$, depends on the investment in the relationship and satisfies $\quad 0 \leq p\left(e_{i, j}\right)<1,0 \leq e_{i, j}<\infty, \quad p(0)=0, \quad\left(\begin{array}{c}\lim P\left(e_{i, j}\right) \\ e_{i, j} \rightarrow \infty\end{array}\right) \rightarrow 1$,

\footnotetext{
${ }^{2}$ Note that while a link is created between two agents both agents bear a cost, but one agent incurs the main cost or the additional cost of maintaining the link. For example, if one individual hosts or phones the second, both individuals have a cost-time, but only one pays for the hospitality or the conversation.
} 


$$
\begin{aligned}
& \left.\frac{\partial p\left(e_{i, j}\right)}{\partial e_{i, j}}>0, \quad \frac{\partial^{2} p\left(e_{i, j}\right)}{\partial e_{i, j}^{2}}<0 \quad \text { (see figure } 1\right) .^{3} \quad \text { For } \quad \text { example, } \\
& p\left(e_{i, j}\right)=1-\exp \left(-\frac{e_{i, j}}{a}\right), a>0 \text { (see figure } 2 \text { for an illustration). }
\end{aligned}
$$

We assume that if there are several paths between two agents, the agents can only benefit from one of the paths, and the agents will choose to connect through the path with the highest intensity (which is not necessarily the shortest path).

Let us define the main structures of social networks: a network is empty if it contains no links, and is complete if a link between every pair of agents exists (see figure 3). Another prominent network structure is a star network, under which all players are linked to one central player and there are no other links (see figure 4).

Denote the optimal effort which the second individual invests in the first individual by $e_{2,1}^{*}$. The second individual forms a link with the first immigrant if, and only if, his benefit is positive, i.e.:

$$
B_{2}=p\left(e_{2,1}^{*}\right) u-e_{2,1}^{*}>0
$$

If equation (1) is not valid then an empty network is obtained. Let us now consider different structures of network formations assuming that equation (1) is satisfied. Denote the effort in a star network by $e^{s}$ and the central agent by 1 . In period $N$ the utility of individual $i \forall i \geq 3$ in a "star network" would be:

$$
B_{i}=p\left(e_{i, 1}^{s}\right)\left(u+\sum_{j=2, j \neq i}^{j=N} p\left(e_{j, 1}^{s}\right) u\right)-e_{i, 1}^{s}
$$

The optimal effort of individual $i$ which is invested in the relationship in the central agent satisfies: ${ }^{3}$ Brueckner (2006), who also assumes that $0 \leq p\left(e_{i, j}\right)<1, \frac{\partial p\left(e_{i, j}\right)}{\partial e_{i, j}}>0$ and $\frac{\partial^{2} p\left(e_{i, j}\right)}{\partial e_{i, j}^{2}}<0$,
explains why this approach can be justified. 


$$
\frac{\partial B_{i}}{\partial e_{i, 1}^{s}}=0 \Rightarrow \frac{\partial p\left(e_{i, 1}^{s}\right)}{\partial e_{i, 1}^{s}}=\frac{1}{u+\sum_{j=2, j \neq i}^{j=N} p\left(e_{j, 1}^{s}\right) u}
$$

\section{Proposition 1:}

In the formation of a "star network", the intensity of the relationships of the agents with the central agent increases every period.

Proof: See Appendix 1.

Lemma 1: If the second and the third individuals form a link with the same individual but not with each other, then all the future agents who arrive will invest in the same individual and a "star network" will be created.

\section{Proof: See Appendix 2.}

In "complete network" each agent forms links with all the previous agents. Thus at period $N$ agent $i$ benefits from $N-1$ direct links, but he maintains the link only with the $i-1$ previous agents. All the agents are identical, hence the agent invests the same effort in them, i.e. $e_{i, 1}^{c}=e_{i, 2}^{c}=\ldots=e_{i, i-1}^{c}$ ( $c$ denotes "complete network").

The utility of individual $i$ is thus given by:

$$
B_{i}=(i-1)\left(p\left(e_{i, 1}^{c}\right) u-e_{i, 1}^{c}\right)
$$

From equation (4) it follows that the optimal effort of the investment of individual $i$ satisfies:

$$
\frac{\partial B_{i}}{\partial e_{i, 1}^{c}}=0 \quad \Rightarrow \quad \frac{\partial p\left(e_{i, 1}^{c}\right)}{\partial e_{i, 1}^{c}}=\frac{1}{u}
$$

As opposed to "star network", the optimal level of effort in the "complete network", $e^{c}$, is constant and is independent of the size of the group. In a "star network" agent $i$ derives benefit from one direct link and $N-2$ indirect links via single investment, 
whereas in a "complete network" the agent benefits from one direct link from each investment. It is easy to see from proposition 1 and figure 1 that as the number of the members in the group increases, the gap between the investments in the two structures increases as well.

By lemma 1, if the third agent forms a link only with one agent - a "star network" is created. Let us assume that the third agent forms a link with the two previous agents. The fourth agent faces three possibilities: (a) to form a link only with one agent and to benefit from one direct link and two indirect links; (b) to form a link with two agents and to benefit from two direct links and one indirect link and (c) to form a link with all the agents and to benefit from three direct links. Denote the optimal investment of the fourth agent by $e_{4, i}^{*}$. With the first possibility, the investment is highest and is equal to the investment in "star network" which is described by (3). In the third possibility the investment equals the investment in “complete network", $e^{c}$. In the second possibility one investment is equal to $e^{c}$ and the second investment satisfies $e^{c}<e_{4, i}^{*}<e_{4, i}^{s}$. The variety of the possibilities for the fifth agent increases and we can obtain different structures of networks.

So far the agents benefit from friends, "friends of friends" and "friends of friends of friends” and so forth. It is clear that benefits decrease smoothly as link distances increase. We can think of situations where the benefits are zero when more than two links are involved (Brueckner, 2006). Let us now examine the network formation where the distance between the links is limited to two.

\section{Proposition 2:}

Under the assumption that the distance between the links is limited to 2, three possible equilibria exist:

1. A "star network" - all individuals invest only in a central agent,

2. A "complete network" - each agent invests in all previous agents,

3. The possibility of moving from a "complete network" to a "star network". At the beginning everyone invests in all previous agents and from a specific agent all agents invest in a single central agent (figure 5).

Proof: See Appendix 3 


\section{Network formation between immigrants and natives}

Suppose now that two sets of individuals join the society at different times, such that time gaps exist between them. Let us call the individuals of the first set "natives" and the individuals of last set "immigrants". ${ }^{4}$ Since immigrants consume specific ethnic goods, we assume that an immigrant can make a link between himself and the natives and between other immigrants and the natives. However immigrants cannot create a linkage between themselves via natives. The connection must be direct and not via natives.

To state matters formally, let

$u_{M L}$ - the utility that an immigrant has from a direct link with a native;

$u_{L M}$ - the utility that a native has from a direct link with an immigrant;

$u_{M M}$ - the utility that an immigrant has from a direct link with an immigrant; Assuming that $u_{M L}>u_{L M}$.

Following Galeotti et al. (2006), we assume that the cost of forming links within the groups is lower as compared to the cost of forming links across groups. Immigration is a salient example as immigrants, in many cases, speak different languages and have different manners than the natives. Hence, if an immigrant wishes to form a link with individuals from the another group, there is a fixed cost, $c$, of acquiring the language and knowing their mentality in addition to the variable cost, $e$. As previously, it is assumed that the agent who gains more benefit from the link bears the cost of the relationship.

Following Berry (1997): Assimilation takes place when the immigrant forms links with natives but severs links with other immigrants. Separation (or segregation) occurs when the immigrant forms links with immigrants but not with natives. Integration happens when the immigrant has interaction with both societies: immigrants and natives. Marginalization takes place when the immigrant fails to maintain interaction with the both societies.

Let us now examine the possible network formations between the natives and the immigrants. The first immigrant faces two main possibilities either to form a link with the natives or not. As shown previously, the natives can be linked as a "star

\footnotetext{
${ }^{4}$ The gap time between the last individual in the first set to the first individual in the last set is sufficient for full assimilation.
} 
network", as a "complete network" or as a different structure. Suppose that the first immigrant chooses the first possibility and forms a link with the natives. Denote the expected benefit of immigrant $i$ from the optimal investments in interactions with the natives by $X_{M_{i}}$, immigrant $i$ by $M_{i}$, native $i$ by $L_{i}$ and the number of natives by $L$. The immigrant benefits from the friendship more than the natives, so he incurs the linking cost. His choice reveals that his net benefit is positive and satisfies:

$$
X_{M_{1}}-\sum_{i=1}^{i=L} e_{M_{1}, L_{i}}-c>0
$$

The second immigrant faces the following possibilities: (a) to form a link only with natives; (b) to form a link only with the first immigrant; (c) to form a link with natives and the first immigrant; and (d) not to form any links at all. From equation (6) and the fact that $X_{M_{1}}=X_{M_{2}}, \sum_{i=1}^{i=L} e_{M_{1}, L_{i}}=\sum_{i=1}^{i=L} e_{M_{2}, L_{i}}$, it is easy to see that when the first immigrant decides to form a link, possibility (d) is not worthwhile for the second immigrant. Let us examine the valid possibilities: Suppose that the immigrant chooses the first possibility and forms a link only with natives. His choice reveals that his net benefit is positive and satisfies:

$$
X_{M_{2}}-\sum_{i=1}^{i=L} e_{M_{2}, L_{i}}-c>X_{M_{2}}-\sum_{i=1}^{i=L} e_{M_{2}, L_{i}}-c+p\left(e_{M_{2}, M_{1}}\right) u_{M M}-e_{M_{2}, M_{1}}
$$

$e_{M_{2}, M_{1}}$ is the optimal investment of the first immigrant in the second immigrant which satisfies $\frac{\partial p\left(e_{M_{2}, M_{1}}\right)}{\partial e_{M_{2}, M_{1}}}=\frac{1}{u_{M M}}$. The LHS of equation (7) presents the net benefit when the immigrant links to natives, whereas the RHS presents the net benefit from linking to natives and the first immigrant. From equation (7) it follows that:

$$
P\left(e_{M_{2}, M_{1}}\right) u_{M M}-e_{M_{2}, M_{1}}<0
$$


This means that the net benefit of an immigrant from the interaction with another immigrant is negative. Since $X_{M_{j}}=X_{M_{2}}, \sum_{i=1}^{i=L} e_{M_{j}, L_{i}}=\sum_{i=1}^{i=L} e_{M_{2}, L_{i}} \forall j \geq 2$, all future immigrants will form a link with the natives but not with other immigrants and a complete assimilation is obtained. Figure 6.1 presents a possible formation of assimilation. The immigrants who are assimilated in the destination country adopt the language, values and manners of the host society and sever their connection with their origin society. This may happen when the social distance between the immigrants and the natives is small and the level of prejudice, ethnocentrism, racism and discrimination is relatively low. Alternatively it occurs when the immigrants are highly skilled and can easily acquire the new language. This result is supported by Bauer, Epstein and Gang (2005), who examined the effect of ethic enclaves on the location choice of Mexicans in the U.S. They found that immigrants with little English proficiency (i.e., their connection cost, $c$, is high) chose to migrate to destinations with large ethnic enclaves and vice versa for immigrants with good English proficiency.

If the second immigrant chooses possibility (b) and links only with the previous immigrant then:

$$
p\left(e_{M_{2}, M_{1}}^{A}\right)\left(u_{M M}+X_{M_{1}}\right)-e_{M_{2}, M_{1}}^{A}>X_{M_{2}}+p\left(e_{M_{2}, M_{1}}^{B}\right) u_{M M}-\sum_{i=1}^{i=L} e_{M_{2}, L_{i}}-c-e_{M_{2}, M_{1}}^{B}
$$

where $e_{M_{2}, M_{1}}^{A}$ is the investment of the second immigrant in the first immigrant, if he forms only a single link and $e_{M_{2}, M_{1}}^{B}$ is the his investment in the first immigrant, this is in addition to links with the natives. As shown above, $e_{M_{j}, M_{1}}^{A} \geq e_{M_{2}, M_{1}}^{A}, X_{M_{1}}=X_{M_{2}}=X_{M_{j}} \quad$ and $\quad e_{M_{j}, M_{k}}^{B} \geq e_{M_{2}, M_{1}}^{B} \forall k \neq 1, j . \quad$ From adding $(j-2)\left(p\left(e_{M_{j}, M_{k}}^{B}\right) u_{M M}-e_{M_{j}, M_{k}}^{B}\right)$ to the both sides of equation (9) and rearranging it we obtain:

$$
\begin{gathered}
p\left(e_{M_{j}, M_{1}}^{A}\right)\left(u_{M M}+X_{M_{1}}\right)-e_{M_{j}, M_{1}}^{A}+(j-2)\left(p\left(e_{M_{j}, M_{k}}^{B}\right) u_{M M}-e_{M_{j}, M_{k}}^{B}\right)> \\
X_{M_{j}}-\sum_{i=1}^{i=L} e_{M_{j}, L_{i}}-c+(j-1)\left(p\left(e_{M_{j}, M_{k}}^{B}\right) u_{M M}-e_{M_{j}, M_{k}}^{B}\right)
\end{gathered}
$$


The LHS of equation (10) presents the net benefit when immigrant $j$ links only to immigrants in the form of a "complete network", whereas the RHS presents the net benefit from linking to the natives and the immigrants (which are connected in the structure of "complete network"). In a similar way, we can show that the inequality, which is presented in (10), is valid not only for "complete networks" but also for each possible structure of immigrants' group (if this structure yields more benefit than the “complete network”). Anyway, from the first two immigrants' behavior we can conclude that all the future immigrants will form a link only with immigrants.

In the case where the second immigrant chooses not to link to the natives, a separation is obtained, i.e. two segregated groups are created: immigrants and natives. Figures 6.2 and 6.3 present possible formations of separation. The unique interaction between these groups is via the first immigrant who is used as a "bridge" between the two societies. The first immigrant is the single agent who incurs the connection cost with the host society and all future immigrants will benefit from his links. We expect that this occurs when the connection cost is high or when the net utility from a link with a native is low, relative to the cost. This would probably hold in the case where immigrants are temporary workers. It should be noted that by proposition 1 and lemma 1, if the third immigrant links only with one immigrant, all the future immigrants will behave like him and a "star network" will be obtained (see figure 6.2). In this case the role of the central agent is double: he is used as a middleman between the immigrants' society and the new society and at the same time is used as a "hub” for the immigrants' society. The intensity of the relationship of immigrants with the first immigrant increases from period to period, and he becomes a more influential leader.

These results are supported by extensive studies on migration networks and assimilation in the host country. For instance, Battu et al. (2004), using the UK Quarterly Labour Force Survey, shows that less assimilated ethnic unemployed workers (i.e., Pakistani and Bangladeshi and Indians) are more likely to utilize family and friendly ties to find a job than more assimilated workers. These assimilated workers use more formal job search methods. Lazear (1999), basing his study on U.S. census data, showed that when the immigrant proportion in the host country increases, their incentive to learn the new culture and acquire the new language decreases. 
Locher (2004) examined immigrants from the F.S.U. to Israel and found that the more educated came earlier. The first immigrants are therefore more skilled, acquire the destination country language and manners, and assimilate in the new country. The next immigrants do not have the incentive to invest any effort in assimilating in the new society and form links only with previous immigrants.

If the second immigrant chooses possibility (c) and forms a link with natives and the first immigrant, the net benefit from a direct link with the immigrant is positive, i.e. $P\left(e_{M_{2}, M_{1}}\right) u_{M M}>e_{M_{2}, M_{1}}$. We can also conclude that:

$$
X_{M_{2}}+p\left(e_{M_{2}, M_{1}}^{B}\right) u_{M M}-\sum_{i=1}^{i=L} e_{M_{2}, L_{i}}-c-e_{M_{2}, M_{1}}^{B}>p\left(e_{M_{2}, M_{1}}^{A}\right)\left(u_{M M}+X_{M_{2}}\right)-e_{M_{2}, M_{1}}^{A}
$$

The LHS presents the second immigrants' benefit from linking to the natives, whereas the RHS presents his benefit from linking only to the first immigrant. The future immigrants will continue to form a link with the natives and the immigrants as long as the net benefit of interaction with natives and immigrants will be higher than the net benefit of interaction with only immigrants. In this scenario, integration is obtained and the immigrants are assimilated in the destination society but maintain a link with their source society. The immigrants acquire the local language and manners but keep their cultural identity. Figures 6.4 and 6.5 present possible formations of this case.

The utility of immigrant $j$ if all the immigrants have a link with immigrant $k$ and he forms a link only with immigrant $k$ is:

$$
B_{M_{j}}=p\left(e_{M_{j}, M_{k}}\right)\left(u_{M M}+X_{M_{k}}+\sum_{i=1, i \neq k}^{i=j-1} p\left(e_{M_{1}, M_{i}}\right) u_{M M}\right)-e_{M_{j}, M_{k}}
$$

Over time the stock of the immigrants in the destination country increases and the expression $\sum_{i=1, i \neq k}^{i=j-1} p\left(e_{M_{1}, M_{i}}\right) u_{M M}$ increases as well. At some point of time, it may be worthwhile for the immigrant to invest only in immigrant $k$ in order to enjoy his links with both immigrants and natives. As shown above, if an immigrant chooses to establish a link with only one immigrant, all future immigrants will follow him and a “star network" will be obtained (see figure 6.6). Regarding the previous immigrants 
who already invested the fixed cost, $c$, they continue to have a link with all the natives but a link with only one immigrant (immigrant $k$ ).

These results are supported by Kahanec (2006) who shows that the disadvantage, of members in minority groups in earnings, increases in size relative to the minority in a region. In our model, we can see that in cases where the number of members in the minority groups is low, the members are assimilated in the new society. However increasing the number of members in the minority groups can cause separation (and as a result, a decrease in wages, (see Chiswick, 1978)).

We summarize the results in the following proposition:

\section{Proposition 3:}

When the first immigrant chooses to establish a link with the natives:

- If the second immigrant also establishes a link with them, but not with the immigrant, we obtain “assimilation”, i.e. all the following immigrants will form a link only with the natives.

- If the second immigrant establishes a link only with the first immigrant, we obtain "separation" (or "segregation"), i.e. all the following immigrants will form a link only with the immigrants.

- If the second immigrant establishes a link with the natives and the first immigrant, we may obtain "integration", i.e. all the next immigrants also link to the natives and to the immigrants. However it is possible to move to "separation" (or "segregation").

We assumed that $u_{M L}>u_{L M}$, thus the immigrant bears the linking cost. However, if all the interactions between the natives and the immigrants are done by a "middleman", as the number of members in the immigrant group increases, the utility of the natives via the indirect links with immigrants increases and we can conclude that:

\section{Proposition 4:}

In the case where only one immigrant forms a link with the natives, when the immigrants stock increases, the natives may maintain the connection and bear the cost (instead of the immigrant).

Moreover, if the structure of the immigrants is a "star network", fewer immigrants are required to bear the linking cost from the immigrant to the natives. 
Proof: Appendix 4.

It should be noted that because of the investment of the natives in the immigrant, the middlemen”, increase as the number of the immigrants in the host country increase (see proposition 1).

Suppose that the first immigrant chooses not to form a link with the natives, thus:

$$
B_{M_{1}}=X_{M_{1}}-\sum_{i=1}^{i=L} e_{M_{1}, L_{i}}-c<0
$$

Since $X_{M_{j}}=X_{M_{2}}, \sum_{i=1}^{i=L} e_{M_{j}, L_{i}}=\sum_{i=1}^{i=L} e_{M_{2}, L_{i}} \forall j \geq 2$, it is clear that the net benefit of the second immigrant from the interaction with natives is also negative, thus he faces only two possibilities: either to form a link with the first immigrant or not at all. If the second immigrant chooses the first possibility then:

$$
B_{M_{2}}=P\left(e_{M_{2}, M_{1}}\right) u_{M M}-e_{M_{2}, M_{1}}>0
$$

As shown above, if the net utility from a link is positive then $B_{M_{j}}>B_{M_{2}} \forall j>2$. From the second immigrant's choice, we can conclude that all the following immigrants will form a link at least with one immigrant. The benefits to the immigrants increase as the number of the immigrants increases. A separation is obtained but, contrary to the previous case, there is no immigrant used as a "bridge" between the two societies. This result could suggest an application for public policy: the government may adopt a policy which decreases the assimilation cost of at least the first immigrants. It will enable interactions between the immigrants and the natives.

Finally, if the second immigrant chooses not to form a link with the first immigrant then:

$$
B_{M_{2}}=P\left(e_{M_{2}, M_{1}}\right) u_{M M}-e_{M_{2}, M_{1}}<0
$$


It is clear that equation (15) is valid for all the following immigrants, thus marginalization is obtained. The immigrants have no (or almost no) interaction with the destination country and the source country. The network formation of the immigrants is an empty network. It should be noted that according to Berry (1997), people rarely choose such an option. Thus,

\section{Proposition 5:}

When the first immigrant chooses not to establish a link with the natives:

- If the second immigrant establishes a link only with the first immigrant, we obtain “separation” (or “segregation"), i.e. all the following immigrants will form a link only with immigrants.

- If the second immigrant chooses also not to establish a link with the immigrants, we obtain "marginalization", i.e. there is no interaction between the immigrants.

\section{Concluding remarks}

In this paper, we analyzed the formation of social networks when the investment in the link is endogenous, i.e. the players choose how much effort to invest in each relationship. Following the pioneering study by Bala and Goyal (2000a) we consider a non-cooperative model of network formation where the cost of link formation is incurred only by the agent who initiates the link. We focus on the intensity of the link in different structures. The paper contributes to the emerging literature which proposes a different approach to the endogenous link strength (see, for example, Brueckner, 2006 and Bloch and Dutta, 2008), however in contrast to this literature, we do not consider an arbitrary number of agents.

Network and social ties play a significant role in the assimilation process (see, for instance, Massey, 1987; Chiswick, 1996; Carrington, 1996; Borjas, 2000 and Bauer et al., 2007). Furthermore, the immigration formation is compatible with the theoretical model: a sequence of homogenous individuals join the country and must decide with whom to form a link. We therefore choose to apply this model to immigration using Berry's (1997) approach. We found that if the two first immigrants form a link with natives but not with each other, assimilation will be achieved. If the first immigrant forms a link with the natives and the second immigrant establishes a link only with the first immigrant, then "separation" will be obtained. The first 
immigrant will be used as a mediator between the host society and the migrants' society. Finally, if the two first immigrants form a link with natives and each other, then "integration” will occur, but moving to "separation” will be possible. The paper supplies evidence from the empirical literature to support these theoretical results.

Our results show that network formations are affected by the first immigrants' behavior which stems from their skills and the social distance between the two societies. Public policy can affect the network formation by the allocation of resources for the assimilation of the first immigrants. Finally, we conclude that if the minority's size is too large, the immigrant, who connects between the immigrants and the natives, will cease to bear the connection cost and the natives will bear this cost instead. This result is interesting because the natives benefit less than the immigrants from the relationship. It should be noted that our model is written as a portrayal of immigrants and natives, but it may be applied in various situations with links across different groups. 


\section{References}

Brueckner, J.K. (2006): “Friendship Networks,” Journal of Regional Science, 46(5), pp. 847-865.

Bala, V. and S. Goyal (2000a): “A Noncooperative Model of Network Formation,” Econometrica, 68(5), 1181-1229.

Bala, V. and S. Goyal (2000b): “A Strategic Analysis of Network Reliability,” Review of Economic Design, 5, 205-228.

Bartel, A.P. (1989): "Where Do the New U.S. Immigrants Live?” Journal of Labor Economics, 7(4), 371-391.

Battu, H, Seaman, P. and Zenou Y. (2004): "Job Contact Networks and the Ethnic Minorities,” CERP Discussion Paper, 5225.

Bauer, T., G.S. Epstein and I.N Gang (2007): "The Influence of Stocks and Flows on Migrants’ Location Choices,” Research in Labor Economics, forthcoming.

Bauer, T., G.S. Epstein and I.N Gang (2005): "Enclaves, Language and the Location Choice of Migrants,” Journal of Population Economics, 18 (4), 649-662.

Berry, J.W. (1997): "Immigration, Acculturation and Adaptation," Applied Psychology, 46(1), 5-68.

Borjas, G.J. (1998): "To Ghetto or not to Ghetto: ethnicity and residential segregation," Journal of Urban Economics, 44, 228-253.

Borjas, G.J. (2000): "Ethnic Enclaves and Assimilation," Swedish Economyc Policy Review, 7, 89-122.

Bloch, F. and Dutta, B (2008): "Communication Networks with Endogenous Link Strength," Games and Economic Behavior, forthcoming.

Carrington, W.J., E. Detragiache and T. Vishwanath, (1996): "Migration with Endogenous Moving Costs,” American Economic Review, 86(4), 909.

Chiswick, B.R. (1978): "The Effect of Americanization on Earning of Foreign-Born Men”, Journal of Political Economy, 86, 897-921.

Chiswick, B.R. and P.M. Miller, (1996): "Ethnic Networks and Language Proficiency among Immigrants”, Journal of Population Economics, 9(1), 19-35.

Chiswick, B.R. and P.M. Miller, (2005): "Do Enclaves Matter in Immigrant Adjustment?”, City and Community, 4 (1), 5-35.

Church, J. and I. King, (1983): "Bilingualism and Network Externalities”, Canadian Journal of Economics, 26(2), 337-45.

Culter, D.M and E.L. Gleaser (1997): “Are Ghettos Good or Bad?”, Quarterly Journal of Economics, 112(3), 827-872.

Currarini, S., O. Jackson and P. Pin (2007): “An Economic Model of Friendship: Homophily, Minorities and Segregation”, DSE Working Paper, No. 20.

Dustmann, C. and I. Preston, (2000): "Racial and Economic Factor in Attitudes to Immigration”, IZA Discussion Paper, No .190, IZA: Bonn.

Echenique, F. and R.G. Fryer Jr. (2007): “A Measure of Segregation Based on Social Interaction,” Quarterly Journal of Economics, 122(2), 441-485.

Goyal, S. and J. Moraga Gonzales (2001): "R\&D networks," RAND Journal of Economics, 32, 686-707.

Galeotti, A, S. Goyal and J. Kamphorst (2006): "Network Formation with Heterogeneous Players”, Games and Economic Behavior, 54, 353-372.

Haller, H. and S. Sarangi (2005): "Nash Networks with Heterogeneous Links," Mathematical Social Sciences, 50, 181-201.

Holzer, H.J. (1987): "Informal Job Search and Black Youth Unemployment”, American Economic Review, 77(3), 446-45. 
Jackson, M.O. (2003): “A Survey of Network Formation: Stability and Efficiency”, Social Science Working Paper, No. 1161.

Jackson, M.O. and A. Wolinsky (1996): “A Strategic Model of Economic and Social Networks”, Journal of Economic Theory, 71(1), 44-74

Jackson, M.O. and A. Watts (2002): "The Evolution of Social and Economics Networks”, Journal of Economic Theory, 106(2), 265-291.

Kahanec, M. (2006): "Ethnic Specialization and Earnings Inequality: Why Being a Minority Hurts but Being a Big Minority Hurts More”, IZA Discussion Paper, No .2050, IZA: Bonn.

Kim, C. and K. Wong (2007): “Network Formation and Stable Equilibrium,” Journal of Economic Theory, 133(1), 536-549.

Lazear, P.E. (1999): “Culture and Language”, Journal of Political Economy, 1074(6), 95-126.

Locher, L. (2004): "Immigration from the Eastern Block and the Former Soviet Union to Israel: Who is Coming When?”, European Economic Review, 48(6), 12431255.

Massey, D.S. (1987): “Understanding Mexican Migration to the United States”, American Journal of Sociology, 92(6).

Montgomery, J.D. (1991): "Social Networks and Labor-Market outcomes: Toward an Economic Analysis”, American Economic Review, 81(5), 1408-1418.

Watts, A. (2001): “A Dynamic Model of Network Formation,” Games and Economic Behavior, 34, 331-341.

Watts, A. (2002): "Non-myopic Formation of Circle Networks", Economics Letters, 74, 277-282. 
Figure 1. The probability of being friends

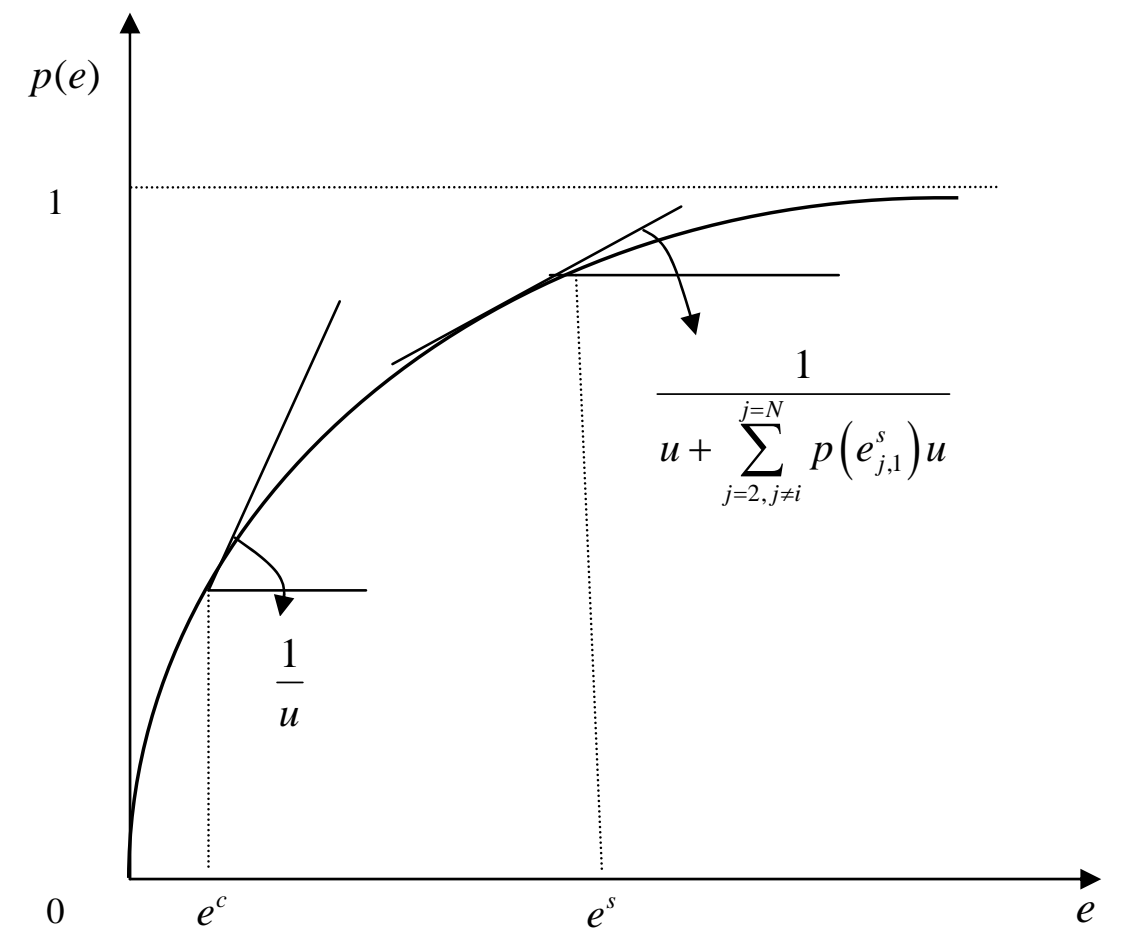

Figure 2. An example

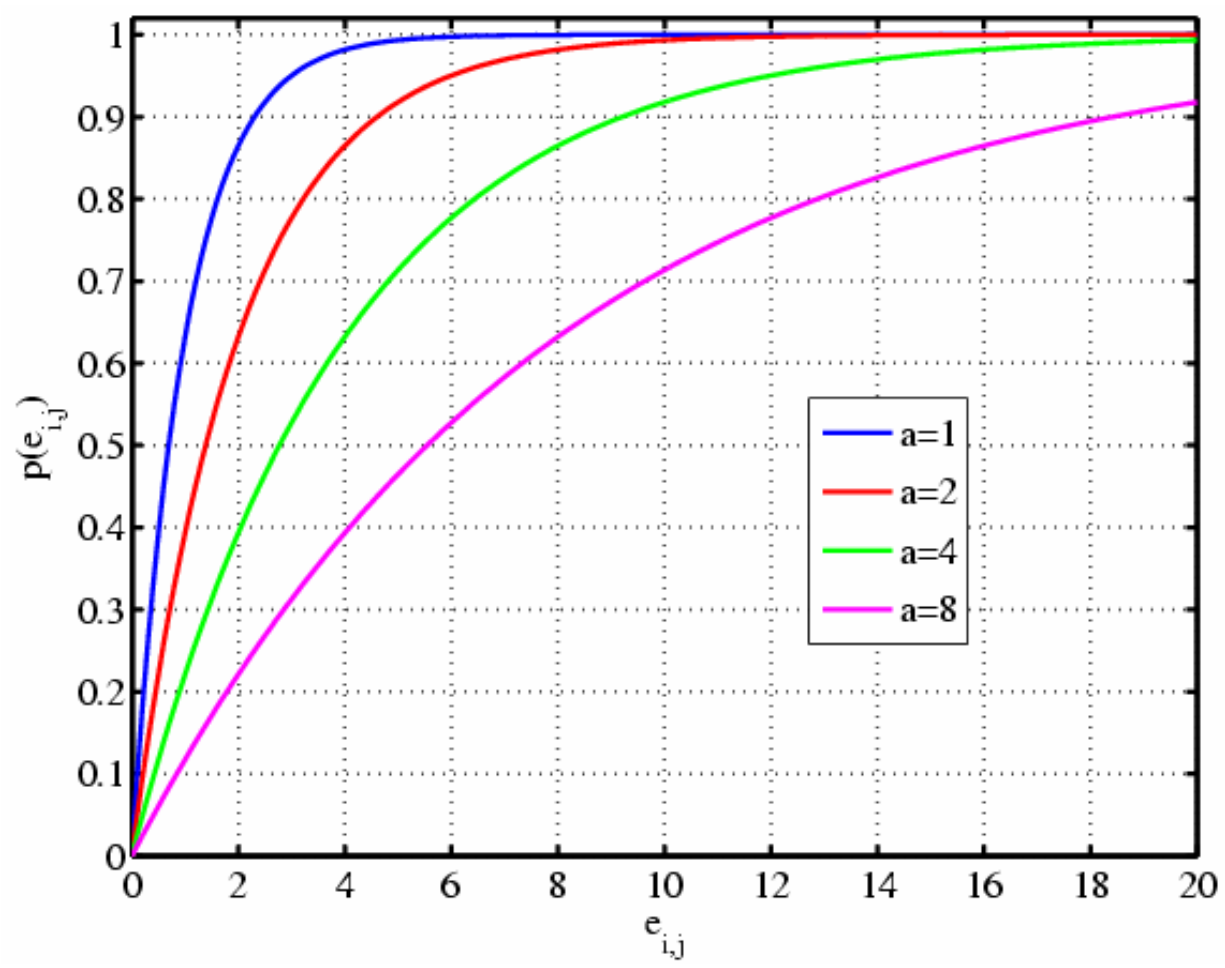


Figure 3. A “complete network”

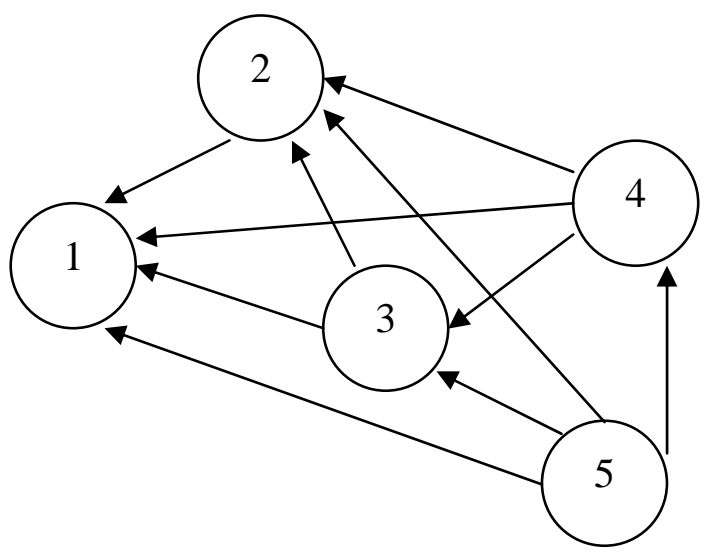

Figure 4. A "star network"

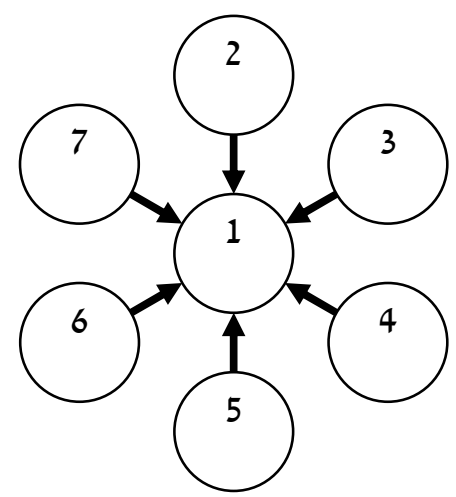

Figure 5. Moving from a "complete network" to a "star network"

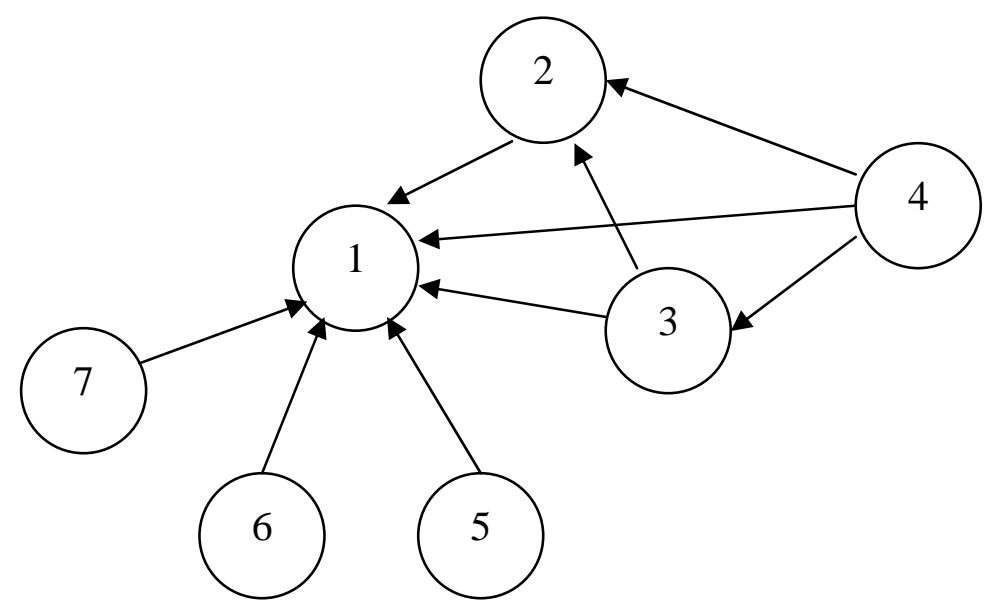


Figure 6. Possible network formations between natives and immigrants

Figure 6.1

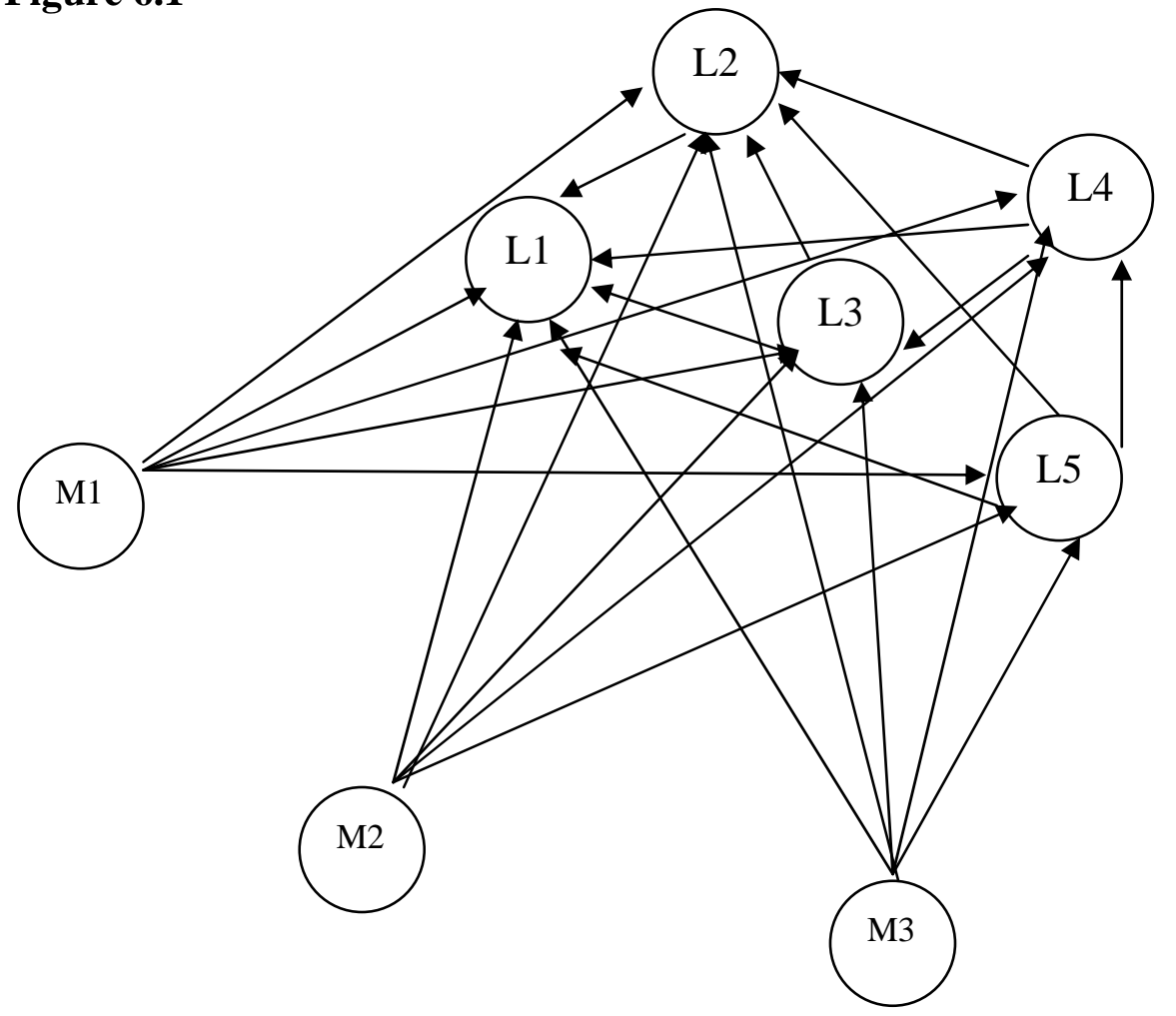


Figure 6.2

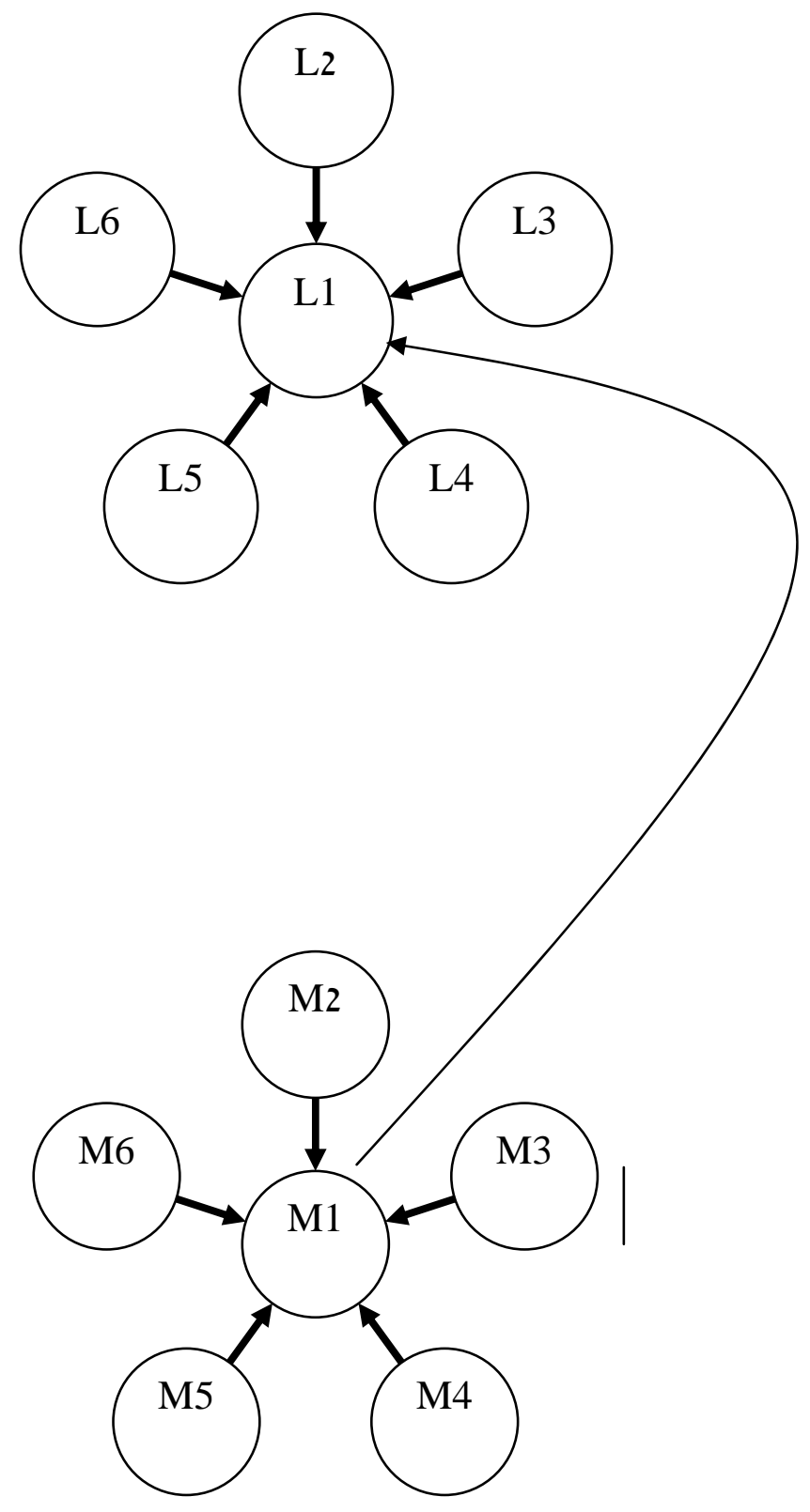


Figure 6.3

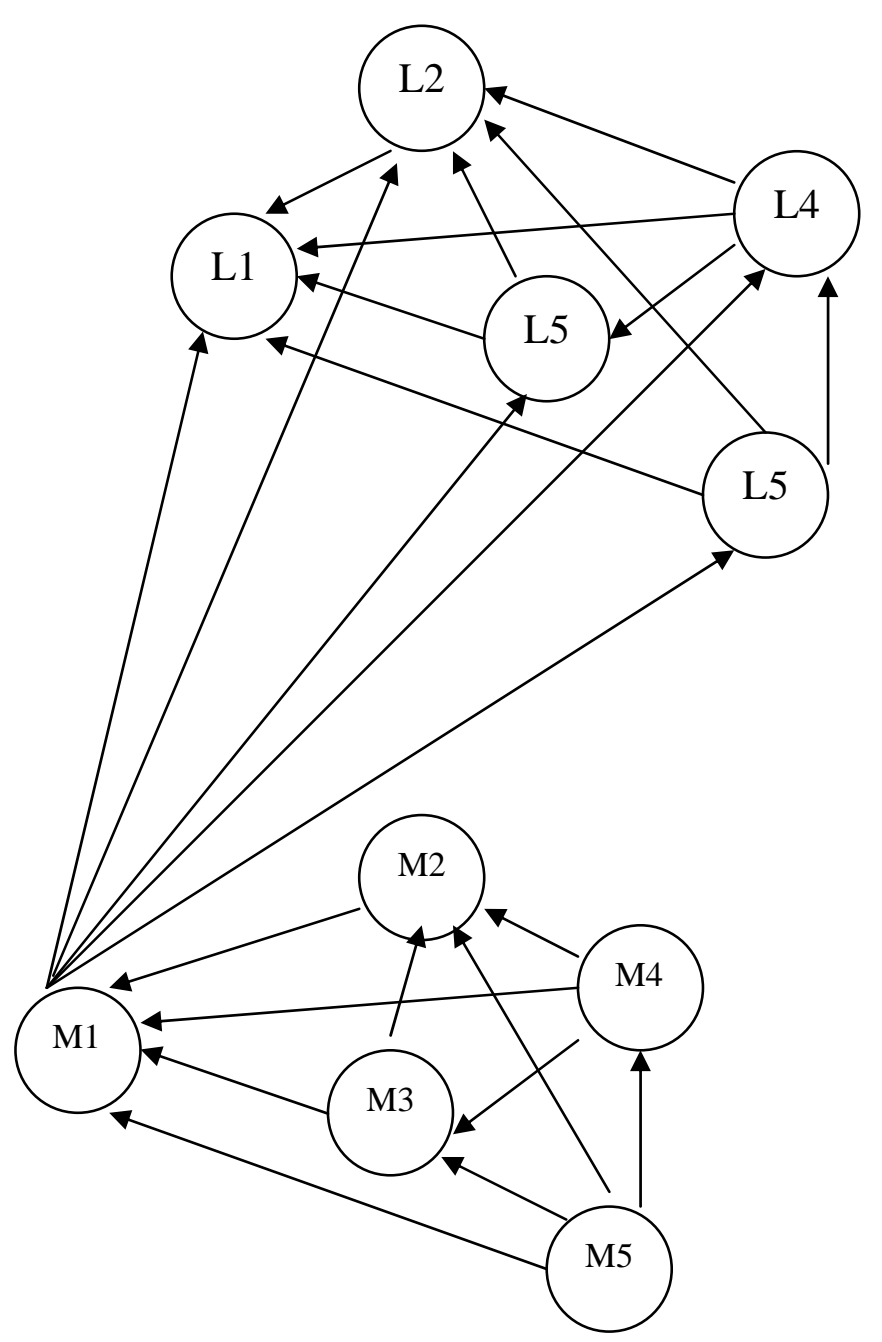

Figure 6.4

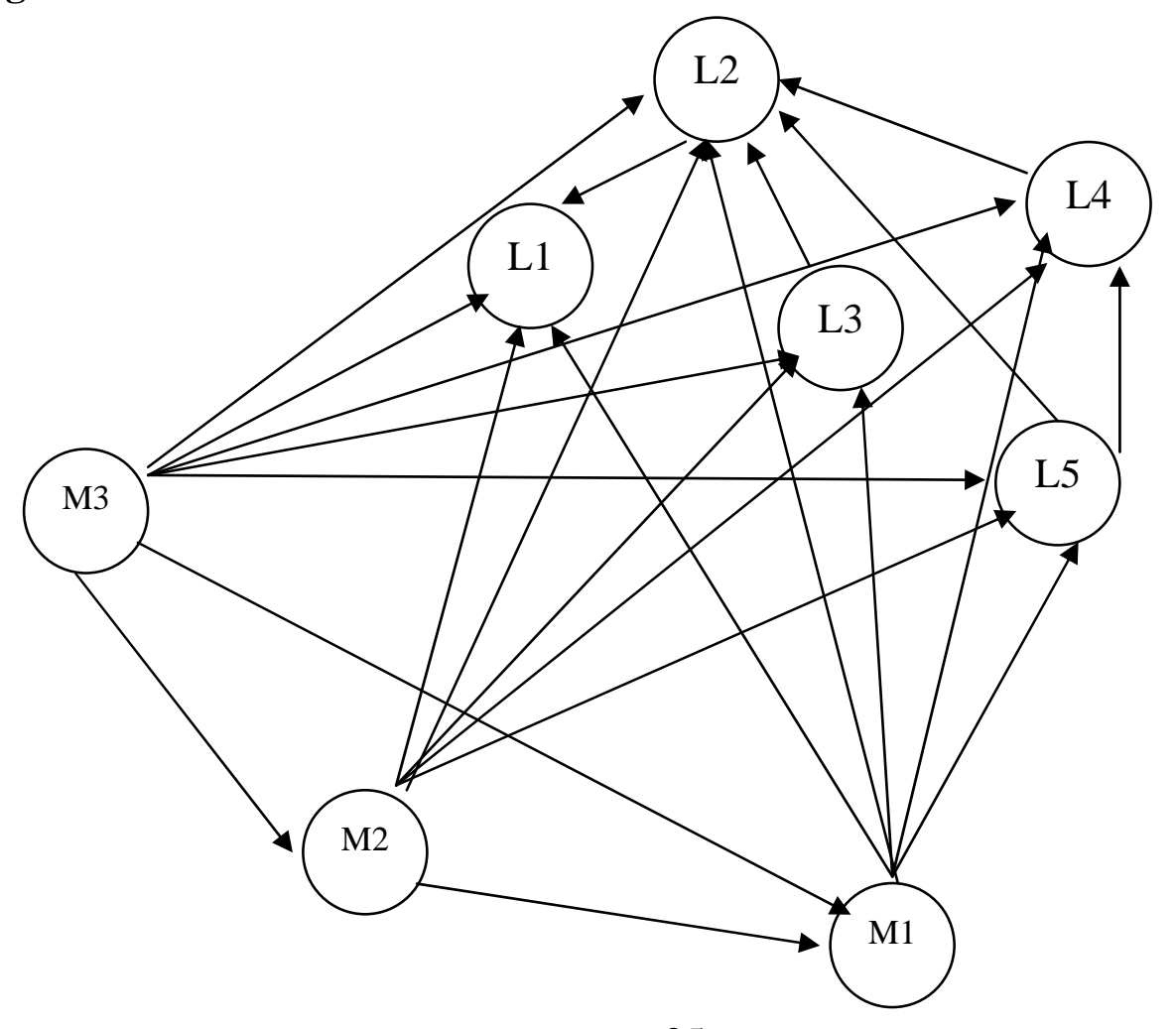




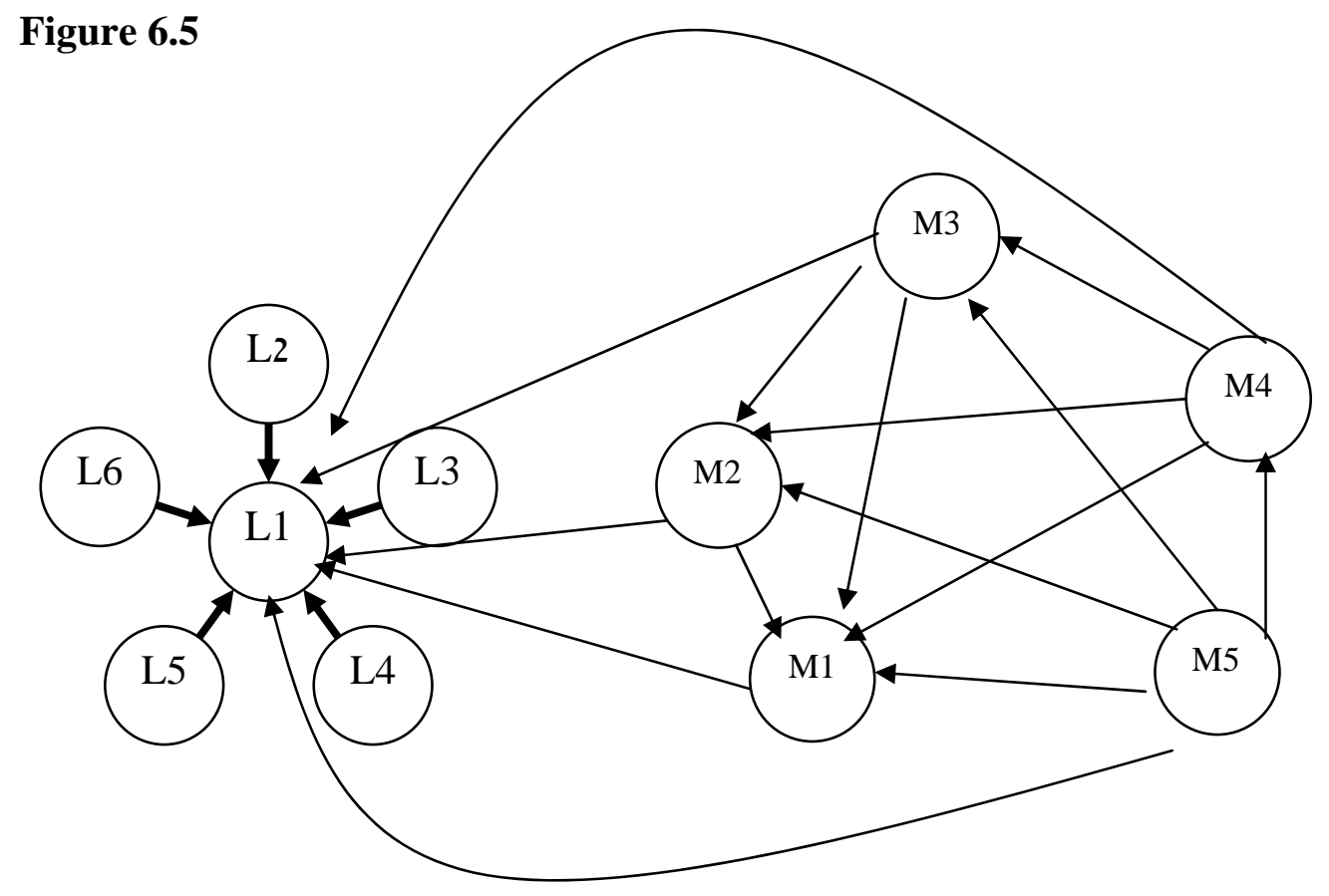

Figure 6.6

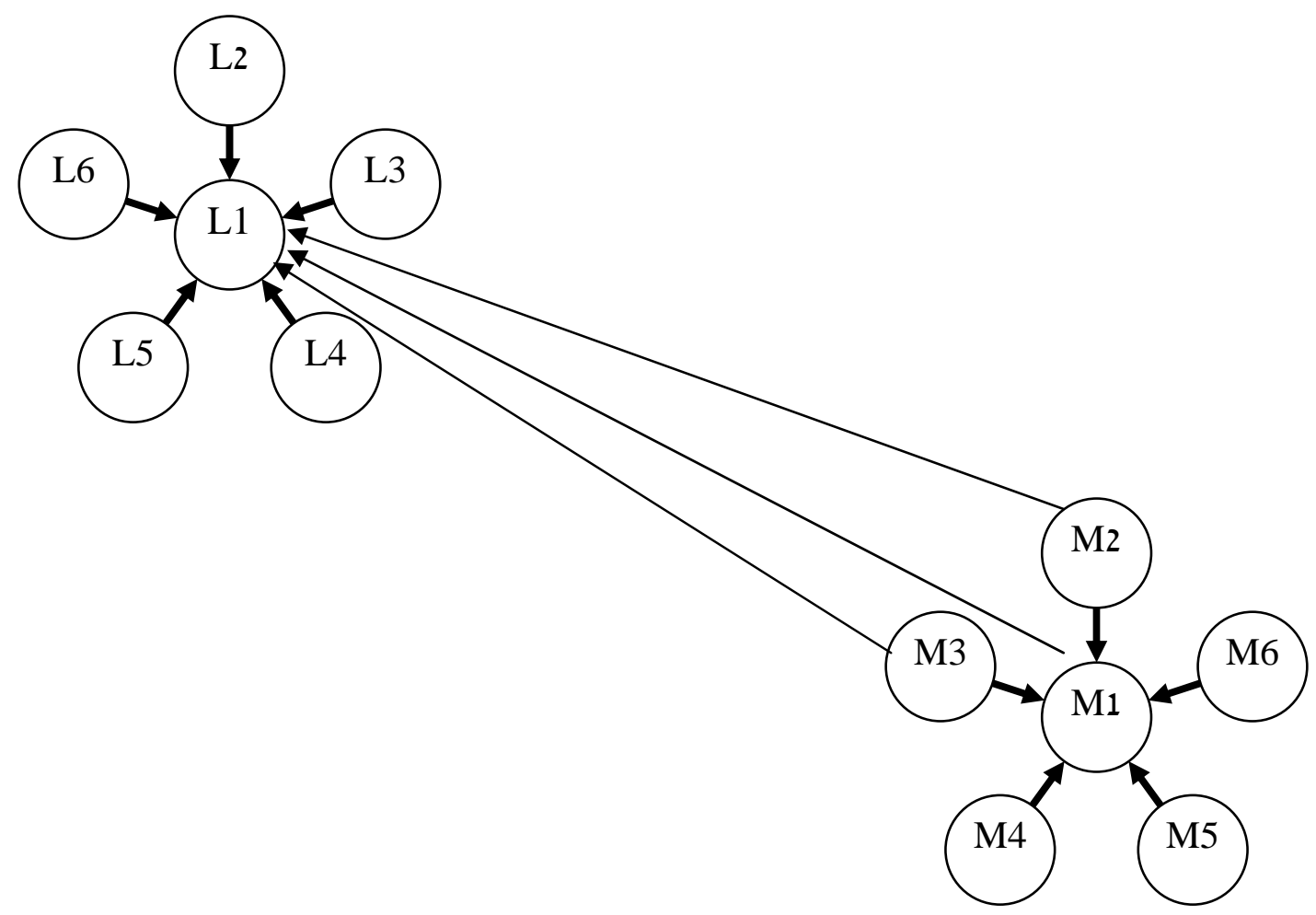




\section{Appendix 1}

In the next period, $N+1$ agents are members in the group, the utility of individual $i$ increases to $B_{i}=p\left(e_{i, 1}^{s}\right)\left(u+\sum_{j=2, j \neq i}^{j=N+1} p\left(e_{j, 1}^{s}\right) u\right)-e_{i, 1}^{s}$, the optimal effort of individual $i$ therefore equals:

$$
\frac{\partial B_{i}}{\partial e_{i, 1}^{s}}=0 \Rightarrow \frac{\partial p\left(e_{i, 1}^{s}\right)}{\partial e_{i, 1}^{s}}=\frac{1}{u+\sum_{j=2, j \neq i}^{j=N+1} p\left(e_{j, 1}^{s}\right) u}
$$

From equations (3) and (16) it follows that:

$$
\frac{1}{u+\sum_{j=2, j \neq i}^{j=N} p\left(e_{j, 1}^{s}\right) u}>\frac{1}{u+\sum_{j=2, j \neq i}^{j=N+1} p\left(e_{j, 1}^{s}\right) u}
$$

$p\left(e_{i, 1}^{s}\right)$ is a concave function. Thus while the slope decreases, the effort increases.

\section{Appendix 2}

Denote the optimal effort of agent $i$ where he benefits from one direct link (which was presented at equation (1)) by $e^{c}$ and his effort if he joins the "star network" by $e_{i}^{S}$. Individual 3 faces two options: the first, to invest in the friendship with one individual (number 1 or 2) and to benefit from an indirect link with another; the second, to invest in both individuals and to benefit from two direct links. If individual 3 decides to invest in only one agent then $B_{3}\left(e_{3}^{s}\right)>B_{3}\left(e^{c}\right)$, hence:

$$
p\left(e_{3}^{s}\right)\left(u+p\left(e^{c}\right) u\right)-e_{3}^{s}>2\left(p\left(e^{c}\right) u-e^{c}\right)
$$

Rearranging equation (18) gives us:

$$
p\left(e_{3}^{s}\right) p\left(e^{c}\right) u>p\left(e^{c}\right) u-e^{c}+\left(p\left(e^{c}\right) u-e^{c}\right)-\left(p\left(e_{3}^{s}\right) u-e_{3}^{s}\right)
$$


$e^{c}$ is the value of $e$ which maximizes $B_{3}\left(e^{c}\right)=p\left(e^{c}\right) u-e^{c}$, thus it is clear that:

$$
p\left(e^{c}\right) u-e^{c}>p\left(e_{3}^{s}\right) u-e_{3}^{s}
$$

Hence, from equation (19) and (20), it follows that:

$$
p\left(e_{3}^{s}\right) p\left(e^{c}\right) u>p\left(e^{c}\right) u-e^{c}
$$

It is assumed that $n-1$ agents are connected in the form of "star network", $e_{n-1}^{s}$ denotes the intensity of their link with the central agent. Let us prove that individual $n$ also connects only to the central agents. Individual $n$ faces a number of options: (a) to form a link with the central agent; (b) to form a link with all the $n-1$ individuals; (c) to form a link with the central agent and with additional $k(0 \leq k \leq n-2)$ agents, and (d) to form a link with $k(0 \leq k \leq n-2)$ agents without the central agent.

From multiplying equation (21) by ( $n-3) \forall i>3$ and adding it to equation (18), the following result is obtained:

$$
p\left(e_{3}^{s}\right)\left(u+(n-2) p\left(e^{c}\right) u\right)-e_{3}^{s}>(n-1)\left(p\left(e^{c}\right) u-e^{c}\right)
$$

$e_{n}^{s}$ is the optimal effort of individual $n$ in the structure of "star network", thus $B_{n}\left(e_{3}^{s}\right)>B_{n}\left(e_{3}^{s}\right)$. If equation (22) is true then:

$$
p\left(e_{n}^{s}\right)\left(u+(n-2) p\left(e_{n-1}^{s}\right) u\right)-e_{n}^{s}>(n-1)\left(p\left(e^{c}\right) u-e^{c}\right)
$$

Thus this means that the benefit of individual $n$ from possibility (a) is higher than the benefit from possibility (b). Now we prove that possibility (a) is better than possibility (c), in order to do it we show that: 
(24)

$p\left(e_{n}^{s}\right)\left(u+(n-2) p\left(e_{n-1}^{s}\right) u\right)-e_{n}^{s}>p\left(e_{n, 1}\right)\left(u+(n-2-k) p\left(e_{n-1}^{s}\right) u\right)-e_{n, 1}+k\left(p\left(e^{c}\right) u-e^{c}\right)$

It is known that $e_{n}^{s}$ is a maximum value of the function level thus:

$$
p\left(e_{n}^{s}\right)\left(u+(n-2) p\left(e_{n-1}^{s}\right) u\right)-e_{n}^{s}>p\left(e_{n, 1}\right)\left(u+(n-2) p\left(e_{n-1}^{s}\right) u\right)-e_{n, 1}
$$

Therefore it is sufficient to proof that:

(26)

$p\left(e_{n, 1}\right)\left(u+(n-2) p\left(e_{n-1}^{s}\right) u\right)-e_{n, 1}>p\left(e_{n, 1}\right)\left(u+(n-2-k) p\left(e_{n-1}^{s}\right) u\right)-e_{n, 1}+k\left(p\left(e^{c}\right) u-e^{c}\right)$

Or, equally:

$$
p\left(e_{n-1}^{s}\right) p\left(e_{n, 1}\right) u>p\left(e^{c}\right) u-e^{c}
$$

By equation (21) and the fact that $p\left(e_{n-1}^{s}\right)>p\left(e_{3}^{s}\right)$ and $p\left(e_{n, 1}\right)>p\left(e^{c}\right)$, equation (27) must be true.

Finally, in order to prove that possibility (a) is better than possibility (d) we show that:

$$
\begin{gathered}
p\left(e_{n}^{s}\right)\left(u+(n-2) p\left(e_{n-1}^{s}\right) u\right)-e_{n}^{s}> \\
p\left(e_{n, i}\right)\left(u+p\left(e_{n-1}^{s}\right) u+(n-3-k) p\left(e_{n-1}^{s}\right) p\left(e_{n-1}^{s}\right) u\right)-e_{n, i}+(k-1)\left(p\left(e^{c}\right) u-e^{c}\right)
\end{gathered}
$$

for $i \neq 1$.

Equation (25) is satisfied, thus in order to prove that equation (28) holds it is sufficient to proof that:

$$
\begin{gathered}
p\left(e_{n, 1}\right)\left(u+(n-2-k) p\left(e_{n-1}^{s}\right) u\right)-e_{n, 1}> \\
p\left(e_{n, i}\right)\left(u+p\left(e_{n-1}^{s}\right) u+(n-3-k) p\left(e_{n-1}^{s}\right) p\left(e_{n-1}^{s}\right) u\right)-e_{n, i}+(k-1)\left(p\left(e^{c}\right) u-e^{c}\right)
\end{gathered}
$$

It is known that $e_{n, 1}$ is a maximum value of the function, thus it is sufficient to proof that: 
(30)

$$
\begin{gathered}
p\left(e_{n, i}\right)\left(u+(n-2-k) p\left(e_{n-1}^{s}\right) u\right)-e_{n, i}> \\
p\left(e_{n, i}\right)\left(u+p\left(e_{n-1}^{s}\right) u+(n-3-k) p\left(e_{n-1}^{s}\right) p\left(e_{n-1}^{s}\right) u\right)-e_{n, i}+(k-1)\left(p\left(e^{c}\right) u-e^{c}\right)
\end{gathered}
$$

By algebraic manipulation we obtain:

$$
p\left(e_{n-1}^{s}\right)<1
$$

Equation (31) is satisfied as assumed above.

\section{Appendix 3}

Individual 3 faces two options: first, to invest in the friendship with one individual and to benefit from an indirect link with another individual, second, to invest in both individuals and to benefit from two direct links. As presented at lemma 1, if individual 3 chooses the first possibly, all future immigrants will follow him and a "star network" will be obtained. Let us now examine the case of a "complete network". If the third individual decides to invest in both agents then $B_{3}\left(e^{c}\right)>B_{3}\left(e_{3}^{s}\right)$, i.e.:

$$
2\left(p\left(e^{c}\right) u-e^{c}\right)>p\left(e_{3}^{s}\right)\left(u+p\left(e^{c}\right) u\right)-e_{3}^{s}
$$

$B_{3}\left(e_{3}^{s}\right)$ is a maximum point, so it is clear that:

$$
p\left(e_{3}^{s}\right)\left(u+p\left(e^{c}\right) u\right)-e_{3}^{s}>p\left(e^{c}\right)\left(u+p\left(e^{c}\right) u\right)-e^{c}
$$

Equations (32) and (33) together give us:

$$
2\left(p\left(e^{c}\right) u-e^{c}\right)>p\left(e^{c}\right)\left(u+p\left(e^{c}\right) u\right)-e^{c}
$$

Or, equally:

$$
p\left(e^{c}\right) u-e^{c}>p\left(e^{c}\right) p\left(e^{c}\right) u
$$

The RHS represents the net benefit from a direct link, whereas the LHS represents the utility from an indirect link. As long as this condition exists, each agent connects to all the previous agents and a "complete network" is created. Now we prove that if this 
condition is not satisfied for individual $i$ then he forms a link only with one individual. The central agent can be each agent who is linked in the network, because the benefit that agent $i$ can yield from each agent (one direct link and $i-2$ indirect links) is identical.

It is given that:

$$
p\left(e_{i}^{s}\right) p\left(e^{c}\right) u>p\left(e^{c}\right) u-e^{c}
$$

We prove that the benefit from one direct link and $i-2$ indirect links is higher than the benefit from $k \forall k>1$ direct links and $i-k-1$ indirect links, it means that:

$$
p\left(e_{i}^{s}\right)\left(u+(i-2) p\left(e^{c}\right) u\right)-e_{i}^{s}>p\left(e_{i}^{*}\right)\left(k u+(i-k-1) p\left(e^{c}\right) u\right)-k e_{i}^{*}
$$

Where $e_{i}^{*}$ is the optimal investment if the individual forms a link with $k$ agents. $e_{i}^{s}$ is the maximum point of the function, it is sufficient to prove that:

$$
p\left(e_{i}^{*}\right)\left(u+(i-2) p\left(e^{c}\right) u\right)-e_{i}^{*}>p\left(e_{i}^{*}\right)\left(k u+(i-k-1) p\left(e^{c}\right) u\right)-k e_{i}^{*}
$$

Rearranging equation (38) gives us:

$$
(1-k) p\left(e_{i}^{*}\right) u-(1-k) e_{i}^{*}>(1-k) p\left(e_{i}^{*}\right) p\left(e^{c}\right) u
$$

Since $1-k<0$, thus:

$$
p\left(e_{i}^{*}\right) p\left(e^{c}\right) u>p\left(e_{i}^{*}\right) u-e_{i}^{*}
$$

By equation (36) and the facts that $e_{i}^{s}>e_{i}^{*}, p\left(e^{c}\right) u-e^{c}>p\left(e_{i}^{*}\right) u-e_{i}^{*}$, equation (40) must be true.

As shown at proposition $1 e_{i+1}^{*}>e_{i}^{*}$. Thus if equation (40) is valid for individual $i$, it will be valid for individual $i+1$. All the future agents will form a link only with the central agent and moving from a "complete network" to a "star network” will be obtained. 


\section{Appendix 4}

Denote the number of the immigrant group members by $M$. The utility which native $j$ obtains from the relationship with immigrant $k$ if the immigrant bears the cost of link is:

$$
B_{M_{k}}=p\left(e_{M_{k}, L_{j}}\right)\left(u_{L M}+\sum_{i=1, i \neq k}^{i=M} p\left(e_{M_{i}, M_{k}}\right) u_{L M}\right)
$$

The benefit of immigrant $k$ from relationship with native $j$ is constant, whereas the utility of the native from this relationship increases during the time. As the stock of immigrants, $M$, increases, the natives benefit from more indirect links with the immigrants. Denote the new number of the immigrants in the host country by $M^{\prime} \forall M^{\prime}>M$. Hence, it is possible that:

$$
p\left(e_{L_{j}, M_{k}}\right)\left(u_{L M}+\sum_{i=1, i \neq k}^{i=M^{\prime}} p\left(e_{M_{i}, M_{k}}\right) u_{L M}\right)-e_{L_{j}, M_{k}}>p\left(e_{M_{k}, L_{j}}\right)\left(u_{L M}+\sum_{i=1, i \neq k}^{i=M} p\left(e_{M_{i}, M_{k}}\right) u_{L M}\right)
$$

Furthermore, if the immigrants are linked by a "star network", the optimal effort which the immigrants invest in the central agent, $e_{M_{i}, M_{k}}$, also increases with the increase in the number of immigrants. The benefit to the native from the relationship with the immigrant, who is the central agent, increases more than if the immigrants are linked in a different structure. Thus fewer immigrants are required in order that equation (42) will be satisfied. 